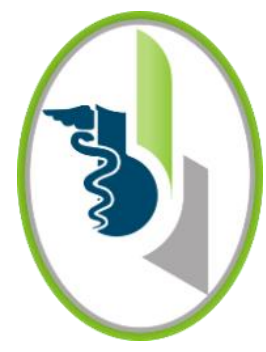

ACCESO $\odot$ ABIERTO

Para citaciones: Lopez, S., Cuadrado, B., Segovia, J. (2019). Resonancia magnética frente a la histopatología en el diagnóstico de neoplasias cerebrales. Revista Ciencias Biomédicas, 8(2), 60-71.

Recibido: 22 de febrero de 2019

Aprobado: 18 de mayo de 2019

Autor de correspondencia:

Sandra López Sierra

sandralucials89@gmail.com

Editor: Inés Benedetti. Universidad de Cartagena-Colombia.

Copyright: (C) 2019. Lopez, S., Cuadrado, B., Segovia, J. Este es un artículo de acceso abierto, distribuido bajo los términos de la licencia https://creativecommons.org/licenses/by-nc-

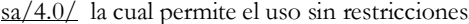
distribución y reproducción en cualquier medio, siempre y cuando el original, el autor y la fuente sean acreditados.

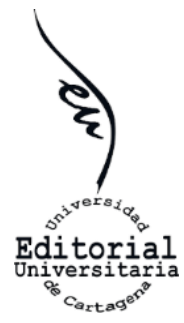

\section{Resonancia magnética frente a la histopatología en el diagnóstico de neoplasias cerebrales}

\author{
Magnetic resonance versus histopathology in the diagnosis of \\ cerebral neoplasms
}

López Sierra Sandra ${ }^{1}$, Cuadrado Cano Bernarda ${ }^{2}$, Segovia Fuentes Javier ${ }^{1}$

${ }^{1}$ Departamento de Radiología e Imágenes Diagnósticas, Facultad de Medicina, Universidad de Cartagena-Colombia
${ }^{2}$ Departamento de Investigaciones. Facultad de Medicina, Universidad de Cartagena-Colombia

\section{RESUMEN}

Introducción: En Colombia se presentan aproximadamente 3,4 casos de neoplasias cerebrales por 100.000 personas-año. En su diagnóstico la resonancia magnética (RM) juega un papel significativo, pues puede aportar información importante al evaluar las lesiones y realizar un planeamiento quirúrgico; por ello, es crucial que sea adecuadamente interpretada y una de las maneras de verificarlo es la correlación radiopatológica.

Objetivo: Identificar el desempeño de la RM utilizada para el diagnóstico de las neoplasias cerebrales de mayor frecuencia en la E.S.E Hospital Universitario del Caribe (H.U.C.) desde 2013 a 2018 y compararla con la histopatología como prueba de oro.

Métodos: Se desarrolló un estudio de prueba diagnóstica en 43 pacientes con neoplasias cerebrales en el H.U.C. Se utilizaron datos del reporte imagenológico, histopatológico y la historia clínica evaluándose variables sociodemográficas, clínicas, imagenológicas y patológicas. Se determinaron diferencias estadísticas entre el diagnóstico radiológico y las demás variables frente a la histopatología. Se determinaron sensibilidad, especificidad, VPP y VPN, cociente de probabilidad positivo y negativo y exactitud global para la RM considerando la histopatología como la prueba de oro.

Resultados: La RM fue más sensible en los casos de meningioma y astrocitoma de bajo grado, fue muy específica para el glioblastoma. En general la RM presentó buenos índices de exactitud diagnóstica. En todos los casos se encontraron diferencias estadísticamente significativas entre la RM y la histopatología.

Conclusiones: En el H.U.C. la RM presenta buen desempeño a la hora de diagnosticar tumores cerebrales, en especial los astrocitomas de bajo grado y los meningiomas y en menor grado para los glioblastomas y las lesiones metastásicas.

Palabras Clave: Imagen por Resonancia Magnética; neoplasias encefálicas; radiología; patología. 


\section{ABSTRACT}

Introduction: In Colombia there are approximately 3.4 cases of brain neoplasms per 100,000 person-years. In the diagnosis, MRI plays a very significant role, since it can provide important information for the treating specialist when evaluating the lesions and performing a surgical planning; therefore, it is crucial that the MRI is properly interpreted and one of the ways to verify it is through the radio-pathological correlation.

Objective: To identify the performance of the MRI for the diagnosis of the most frequent cerebral neoplasms in the H.U.C from 2013 to 2018 and to compare it with the histopathology as a gold standard.

Methods: A diagnostic test study was performed in 43 patients with more frequent brain neoplasms in the H.U.C. We used the data from the imaging report, the histopathological report and the clinical history evaluating sociodemographic, clinical, imaging and pathological variables. The existence of statistical differences between radiological and histopathological diagnosis and among the other variables with respect to histopathology, as well as sensitivity, specificity, PPV and NPV, positive and negative likelihood ratio and overall accuracy were determined for MRI considering histopathology as a gold standard.

Results: MRI was more sensitive in cases of meningioma and low-grade astrocytoma, while it was very specific for glioblastoma. In general, MRI showed good rates of diagnostic accuracy. In all cases, Fisher's exact test was applied and it was found that there are statistically significant differences between MRI and histopathology.

Conclusion: In the H.U.C., MRI shows good performance when diagnosing brain tumors, especially low-grade astrocytomas and meningiomas and to a lesser degree for glioblastomas and metastatic lesions

Key Words: Magnetic Resonance Imaging; brain neoplasms; radiology; pathology. 


\section{INTRODUCCIÓN}

Según la Organización Mundial de la Salud (OMS) el cáncer es una de las principales causas de morbilidad en el mundo y constituye la segunda causa de muerte (1). Dentro de este grupo de patologias, el cáncer del Sistema Nervioso Central (SNC) ocupa el puesto número 16 con una tasa de incidencia de 6.4 casos por 100.000 habitantes por año (2). En Colombia según los registros del Instituto Nacional de Cancerología, la tasa de incidencia de neoplasias del SNC es de 3,4 por 100.000 personas-año en hombres y de 2,5 por 100.000 personas-año en mujeres (3). En la ciudad de Cartagena en el año 2011 los tumores cerebrales ocuparon el décimo lugar en cuanto a mortalidad por cáncer (4) y, en un estudio realizado entre el 2001 y el 2006 se encontró una frecuencia de tumores primarios del SNC más elevada en esta ciudad que en el resto del país, con una tasa de incidencia general de 6,91 por 100.000 personas año (5).

Para la clasificación de las neoplasias del SNC, se utiliza ampliamente el esquema propuesto por la OMS que engloba hallazgos histopatológicos, inmunohistoquímicos y moleculares, los cuales proporcionan al especialista tratante un diagnóstico definitivo y pronóstico esperado (6).

Es de resaltar que, si bien la prueba de oro la constituye la histopatología, esto supone someter al paciente a una intervención quirúrgica y retrasar el diagnóstico durante el tiempo que regularmente tarda en procesarse la muestra, por lo que las neuroimágenes ganan importancia debido a que al ser estudios no invasivos contribuyen a mejorar la oportunidad diagnóstica y en particular el planeamiento quirúrgico.

Las imágenes por Resonancia Magnética (RM) debido a los principios físicos y técnicos que utilizan se han constituido como la técnica más útil y de elección en pacientes con neoplasias cerebrales (7). Con base en las imágenes que se registran y la información que dispone en el momento, el radiólogo brinda su opinión profesional y sugiere un posible diagnóstico; sin embargo, cuando no se dispone de información adicional, es posible encontrar discrepancias en cuanto a la opinión que proporcionó el especialista.

En este sentido, dentro de los indicadores de resultado de un servicio de radiología se encuentra el de la validez y confiabilidad diagnóstica y una de las maneras de cuantificarla es mediante la correlación radioquirúrgica y radiopatológica (8), en este caso particular, en los pacientes con neoplasias cerebrales establecer la correlación entre la RM y el diagnóstico histopatológico, considerada esta como la prueba definitiva o de oro.

Adicionalmente, previo a cualquier tipo de intervención neuroquirúrgica, como es el caso de las neoplasias cerebrales se debe tener una imagen y por lo tanto, el registro del correspondiente reporte radiológico da una idea preliminar al médico tratante sobre cómo debe encaminar el tratamiento. En pacientes con antecedentes familiares importantes o con mayor riesgo de desarrollar neoplasias cerebrales, la RM también contribuirá a detectar lesiones en estadios iniciales mucho antes de que se presenten síntomas clínicos. De acuerdo a lo anterior, si la RM no es congruente con la histopatología, en consecuencia, se puede subdiagnosticar al paciente y demorar el tratamiento, lo que puede aumentar la mortalidad o las secuelas funcionales, sin olvidar el impacto económico sobre el paciente y las instituciones de salud no realizar una detección oportuna.

Existen algunos estudios retrospectivos reportados a nivel mundial que hacen una correlación radiopatológica comparando la RM con la histopatología de diferentes tipos de tumores cerebrales, entre ellos están: Noguchi et al. en el 2008 (9) con diferentes tumores cerebrales y entre gliomas de alto y bajo grado; en Cuba Rodríguez Pino y cols. en el 2010 (10) en pacientes con tumores primarios y secundarios; y en México Saldívar y cols. en el 2016 (11) en pacientes con tumores astrocíticos. En Colombia los estudios encontrados solo se enfocan en mostrar el comportamiento tanto epidemiológico como clínico de esta patología, dentro de estos tenemos a Ramos y cols., en el 2010 
(5) en la ciudad de Cartagena; Paez-Rodríguez y cols., en el 2013 (12) en la ciudad de Pereira; VegaMolina en el 2016 (13) en la ciudad de Bogotá; por lo cual, dadas las condiciones propias a los equipos y personal encargado de la realización e interpretación de los estudios en el H.U.C. es necesario identificar las diferencias entre las dos metodologías, evaluar la calidad de los informes radiológicos y en consecuencia, evidenciar.

Teniendo en cuenta que existen unos patrones imagenológicos descritos para las neoplasias cerebrales de mayor prevalencia, y que existe un vacío en el conocimiento relacionado con el campo de validez y confiabilidad de la RM que se ha venido utilizando para hacer el diagnóstico de las neoplasias cerebrales de mayor prevalencia a nivel regional, el objetivo del presente estudio fue identificar el desempeño de la RM utilizada para el diagnóstico de las neoplasias cerebrales de mayor frecuencia en el H.U.C. desde 2013 a 2018 y compararla con la histopatología como prueba de oro.

\section{MÉTODOS}

Se desarrolló un estudio de prueba diagnóstica de los casos de neoplasias cerebrales con mayor frecuencia en los registros del H.U.C. desde octubre de 2013 hasta marzo de 2018, que cumplieran con todos los criterios de inclusión. Se utilizaron los datos del reporte imagenológico, del informe histopatológico y de la historia clínica de los pacientes con diagnóstico de tumor cerebral. Los criterios de inclusión fueron: historias de pacientes de cualquier edad que en el reporte de RM una opinión o una descripción compatible con neoplasia cerebral y que además se les realizado estudio histopatológico completo.

Teniendo en cuenta las proyecciones poblacionales del DANE para Cartagena y con una incidencia de tumores del SNC en la ciudad de 6,9 casos por 100.000 habitantes (5) encontramos una población total de 71 pacientes; basado en esto se calculó con un nivel de confianza del $95 \%$ y un porcentaje de error del 5\%, una muestra de 42 pacientes. Para el cálculo de la muestra se utilizó el software OPEN -
EPI Open Source Epidemiologic Statistics for Public Health versión 3.01, de acceso libre (https://www.openepi.com/Menu/OE_Menu.htm).

Se realizó una búsqueda en la base de datos de patología del H.U.C. de los casos que se presentaron durante el tiempo del estudio, con los descriptores: tumor cerebral, cerebro, cerebelo, fosa posterior, meningioma, astrocitoma, glioblastoma, glioma, metástasis, meduloblastoma y oligodendroglioma, con lo cual se encontró un total de 106 casos. Posteriormente se cruzó la información con la base de datos de radiología del H.U.C. para establecer a cuántos de ellos se les había realizado RM, encontrándose un total de 69 casos, de los cuales 50 cumplieron con los criterios de inclusión y dado que se seleccionaron solo los cuatro tipos de tumores más frecuentes (meningioma, glioblastoma, metástasis y astrocitoma de bajo grado), el estudio incluyó 43 pacientes en total.

Se analizaron los siguientes grupos de variables: sociodemográficas (edad, género, residencia), clínicas (manifestaciones clínicas, tiempo de aparición de los síntomas), imagenológicas (comportamiento en T1, T2, FLAIR, DWI y con el contraste, cantidad y localización de las lesiones, presencia de edema perilesional, componente quístico, calcificaciones, diagnóstico imagenológico) y patológicas (diagnóstico definitivo). La información fue recolectada a partir de los reportes histopatológicos, de resonancia magnética y las historias clínicas en un formato de recolección de datos construyéndose una base de datos en hoja electrónica de Excel (Microsoft Office 2016 bajo Windows $8.1^{\circledR}$ ), previa autorización de la institución.

\section{Análisis estadístico}

Se realizó inicialmente un análisis descriptivo, univariado donde se determinó la normalidad y a partir de ello, las medidas de tendencia central para las variables cuantitativas con sus respectivas medidas de dispersión y para las variables cualitativas, la frecuencia absoluta y relativa. Según la normalidad de las variables cuantitativas analizada mediante la prueba de Shapiro-Wilk, se 
procedió a establecer la existencia o no de diferencias estadísticamente significativas entre los dos diagnósticos: el radiológico y el histopatológico $\mathrm{y}$, entre las variables sociodemográficas, clínicas, distribución en el tiempo frente a la histopatología mediante la aplicación de Chi cuadrado o prueba exacta de Fisher para variables cualitativas. Se determinaron la sensibilidad, especificidad, VPP y VPN, cociente de probabilidad positivo y negativo y exactitud global para la RM considerando la prueba histopatológica como la prueba de Oro tanto a nivel global como por tipo de tumor de mayor prevalencia encontrado.

Se usaron intervalos de confianza del $95 \%$ y se consideró estadísticamente significativo un valor de $\mathrm{p}<0,05$. Para el análisis se utilizó el software IBM SPSS Statistics versión 22.0.

\section{RESULTADOS}

Se obtuvo un total de 43 casos de pacientes con tumores cerebrales, en donde el 53,5\% $(n=23)$ fue de sexo femenino; el 97,7\% $(n=42)$ provenientes del departamento de Bolívar, siendo el 76,7 \% $(n=33)$ residentes en el área urbana (Cartagena) y el 23,3\% $(\mathrm{n}=10)$ en el área rural. Para la edad se encontró un promedio de 56,7 años (DS $\pm 13,79$ ). Las manifestaciones clínicas al inicio de la enfermedad más frecuentes fueron cefalea en el 32,6\% $(n=14)$ y convulsiones en el 32,6\% $(n=14)$. Al analizar el tiempo transcurrido entre el inicio de síntomas y la consulta al H.U.C. se encontró que el 53,5 \% $(n=23)$ de los pacientes consultaron en los primeros 30 días, $41,9 \%(n=18)$ entre 2 meses y 1 año y el $4,6 \%(n=2)$ después de1 año (Tabla 1).

De acuerdo a los resultados de la histopatología el $30,2 \%(n=13)$ de los tumores fueron meningiomas, $27,9 \%(\mathrm{n}=12)$ glioblastomas multiformes, $25,6 \%$ $(n=11)$ lesiones metastásicas y $16,3 \% \quad(n=7)$ astrocitomas de bajo grado. En el 39,6\% $(n=17)$ de los casos en el reporte de la resonancia magnética, fue diagnosticado como meningioma, el 23,3\% $(\mathrm{n}=10)$ lesiones metastásicas, el $16,3 \% \quad(\mathrm{n}=7)$ gliomas de bajo grado, 18,6\% $(\mathrm{n}=8)$ astrocitomas de bajo grado y $13,9 \%(n=6)$ glioblastoma multiforme. En dos de los casos no se reportó tumor, de estos uno correspondió a isquemia y el otro (Tabla 1).

Los síntomas más frecuentes por tipo de tumor fueron cefalea $(32,6 \%)$ y convulsiones $(32,6 \%)$ para los cuatro tipos histopatológicos de tumor, sin encontrarse diferencias estadísticamente significativas entre estos $(p=0,932)$ (Tabla 2$)$.

Del total de tumores $23,3 \%(n=10)$ ocurrieron en el grupo de edad de 60 a 69 años, seguido del 20,9\% $(n=9)$ entre 50 a 59 años y 20,9\% (n=9) entre 40 a 49 años; al hacer la comparación por tipo histológico, se encontró que la totalidad de los casos de astrocitoma de bajo grado se presentaron entre los 20 y los 49 años, mientras que los casos de glioblastoma y meningioma estaban entre los $40 \mathrm{y}$ 79 años encontrándose diferencias estadísticamente significativas $(\mathrm{p}<0,05)($ Tabla 3$)$.

En cuanto a la distribución de los tumores por género, el astrocitoma de bajo grado se presentó más en hombres $(57,1 \%)$, el glioblastoma más en mujeres $(83,3 \%)$, el meningioma fue ligeramente más frecuente en mujeres $(53,8 \%)$ y las metástasis en los hombres $(72,7 \%)$ sin diferencias estadísticamente significativas $(p=0,054)($ Tabla 4$)$.

Según la distribución en el tiempo, la mayoría de los casos $(83,7 \%)$ se presentaron entre el año 2014 y el 2017, encontrándose que la frecuencia más alta se presentó con el meningioma durante los años 2014 (40\%), 2016 (37,5\%) e inicios del 2018 (66,7\%) (Tabla 5). 
Tabla 1. Características sociodemográficas, clínicas y paraclínicas de los pacientes objeto de estudio. $n=43$

\begin{tabular}{|c|c|c|}
\hline $\begin{array}{c}\text { CARACTERÍSTICAS SOCIODEMOGRÁFICAS, } \\
\text { CLIINICAS Y PARACLÍNICAS }\end{array}$ & $\mathbf{n}$ & $\%$ \\
\hline Edad & $56,7(\mathrm{DS} \pm 13,79)$ & \\
\hline \multicolumn{3}{|l|}{ Género } \\
\hline $\mathrm{F}$ & 23 & 53,5 \\
\hline M & 20 & 46,5 \\
\hline \multicolumn{3}{|l|}{ Departamento } \\
\hline Bolívar & 42 & 97,7 \\
\hline Córdoba & 1 & 2,3 \\
\hline \multicolumn{3}{|l|}{ Zona de Residencia } \\
\hline Urbana & 33 & 76,7 \\
\hline Rural & 10 & 23,3 \\
\hline \multicolumn{3}{|l|}{ Manifestaciones clínicas } \\
\hline Cefalea & 14 & 32,6 \\
\hline Convulsiones & 14 & 32,6 \\
\hline Pérdida de la fuerza & 6 & 14,0 \\
\hline Hemiparesia & 4 & 9,3 \\
\hline Pérdida de la visión & 2 & 4,7 \\
\hline Disartria & 1 & 2,3 \\
\hline Síntomas constitucionales & 1 & 2,3 \\
\hline Vértigo & 1 & 2,3 \\
\hline \multicolumn{3}{|l|}{ Tiempo de síntomas } \\
\hline 1 a 7 días & 14 & 32,6 \\
\hline 8 a 15 días & 5 & 11,6 \\
\hline 16 a 30 días & 4 & 9,3 \\
\hline 2 a 6 meses & 15 & 34,9 \\
\hline 7 a 12 meses & 3 & 7,0 \\
\hline$>1$ año & 2 & 4,6 \\
\hline \multicolumn{3}{|l|}{ Patología } \\
\hline Meningioma & 13 & 30,2 \\
\hline Glioblastoma & 12 & 27,9 \\
\hline Metástasis & 11 & 25,6 \\
\hline Astrocitoma de bajo grado & 7 & 16,3 \\
\hline \multicolumn{3}{|l|}{ Diagnóstico imagenológico } \\
\hline Meningioma & 17 & 39,6 \\
\hline Metástasis & 10 & 23,3 \\
\hline Astrocitoma de bajo grado & 8 & 18,6 \\
\hline Glioblastoma & 6 & 13,9 \\
\hline Abscesos & 1 & 2,3 \\
\hline Isquemia & 1 & 2,3 \\
\hline \multicolumn{3}{|l|}{ Presencia de lesión tumoral en imagen } \\
\hline $\mathrm{Si}$ & 41 & 95,4 \\
\hline No & 2 & 4,6 \\
\hline
\end{tabular}


Tabla 2. Manifestaciones clínicas vs diagnóstico histopatológico de los pacientes objeto de estudio, $n=43$

HISTOPATOLOGÍA

\begin{tabular}{|c|c|c|c|c|c|c|c|c|c|c|}
\hline \multirow[b]{2}{*}{$\begin{array}{l}\text { MANIFESTACIONES } \\
\text { CLIINICAS }\end{array}$} & \multicolumn{2}{|c|}{$\begin{array}{l}\text { Astrocitoma de } \\
\text { bajo grado }\end{array}$} & \multicolumn{2}{|c|}{ Glioblastoma } & \multicolumn{2}{|c|}{ Meningioma } & \multicolumn{2}{|c|}{ Metástasis } & \multicolumn{2}{|c|}{ Total } \\
\hline & n & $\%$ & $\mathbf{n}$ & $\%$ & $\mathbf{n}$ & $\%$ & $\mathbf{n}$ & $\%$ & $\mathbf{n}$ & $\%$ \\
\hline Cefalea & 2 & 4,7 & 4 & 9,3 & 3 & 7,0 & 5,0 & 11,6 & 14,0 & 32,6 \\
\hline Convulsiones & 3 & 7,0 & 4 & 9,3 & 4 & 9,3 & 3,0 & 7,0 & 14,0 & 32,6 \\
\hline Pérdida de la fuerza & 1 & 2,3 & 2 & 4,7 & 2 & 4,7 & 1,0 & 2,3 & 6,0 & 14,0 \\
\hline Hemiparesia & 1 & 2,3 & 1 & 2,3 & 0 & 0,0 & 2,0 & 4,7 & 4,0 & 9,3 \\
\hline Pérdida de la visión & 0 & 0,0 & 1 & 2,3 & 1 & 2,3 & 0,0 & 0,0 & 2,0 & 4,7 \\
\hline Disartria & 0 & 0,0 & 0 & 0,0 & 1 & 2,3 & 0,0 & 0,0 & 1,0 & 2,3 \\
\hline Síntomas constitucionales & 0 & 0,0 & 0 & 0,0 & 1 & 2,3 & 0,0 & 0,0 & 1,0 & 2,3 \\
\hline Vértigo & 0 & 0,0 & 0 & 0,0 & 1 & 2,3 & 0,0 & 0,0 & 1,0 & 2,3 \\
\hline Total & 7 & 16,3 & 12 & 27,9 & 13 & 30,2 & 11,0 & 25,6 & 43,0 & 100,0 \\
\hline
\end{tabular}

Tabla 3. Frecuencia de acuerdo a edad de las neoplasias cerebrales en el H.U.C. diagnosticadas por histopatología, a nivel global y por tipo de lesión, $n=43$

\section{HISTOPATOLOGIA}

\begin{tabular}{lcccccccccc}
\hline & \multicolumn{2}{c}{$\begin{array}{c}\text { Astrocitoma de bajo } \\
\text { grado }\end{array}$} & \multicolumn{2}{c}{ Glioblastoma } & \multicolumn{2}{c}{ Meningioma } & \multicolumn{2}{c}{ Metastasis } & \multicolumn{2}{c}{ Total } \\
\hline EDAD & $\mathbf{n}$ & $\mathbf{\%}$ & $\mathbf{n}$ & $\mathbf{\%}$ & $\mathbf{n}$ & $\mathbf{\%}$ & $\mathbf{n}$ & $\mathbf{\%}$ & $\mathbf{n}$ & $\mathbf{\%}$ \\
\hline 20 a 29 & 1 & 14,3 & 0 & 0,0 & 0 & 0,0 & 0 & 0,0 & 1 & 2,3 \\
\hline 30 a 39 & 4 & 57,1 & 0 & 0,0 & 0 & 0,0 & 1 & 9,1 & 5 & 11,6 \\
\hline 40 a 49 & 2 & 28,6 & 1 & 8,3 & 5 & 38,5 & 1 & 9,1 & 9 & 20,9 \\
\hline 50 a 59 & 0 & 0,0 & 3 & 25,0 & 3 & 23,1 & 3 & 27,3 & 9 & 20,9 \\
\hline 60 a 69 & 0 & 0,0 & 5 & 41,7 & 3 & 23,1 & 2 & 18,2 & 10 & 23,3 \\
\hline 70 a 79 & 0 & 0,0 & 3 & 25,0 & 2 & 15,4 & 2 & 18,2 & 7 & 16,3 \\
\hline 80 a 89 & 0 & 0,0 & 0 & 0,0 & 0 & 0,0 & 2 & 18,2 & 2 & 4,7 \\
\hline Total & 7 & 100,0 & 12 & 100,0 & 13 & 100,0 & 11 & 100,0 & 43 & 100,0 \\
\hline
\end{tabular}

Tabla 4. Frecuencia de acuerdo al género de las neoplasias cerebrales en el H.U.C. diagnosticadas por histopatología tanto a nivel global como por tipo de lesión, $n=43$

\section{HISTOPATOLOGIA}

\begin{tabular}{|c|c|c|c|c|c|c|c|c|c|c|}
\hline \multirow[b]{2}{*}{ GÉNERO } & \multicolumn{2}{|c|}{$\begin{array}{c}\text { Astrocitoma de } \\
\text { bajo grado }\end{array}$} & \multicolumn{2}{|c|}{ Glioblastoma } & \multicolumn{2}{|c|}{ Meningioma } & \multicolumn{2}{|c|}{ Metástasis } & \multicolumn{2}{|c|}{ Total } \\
\hline & $\mathbf{n}$ & $\%$ & $\mathbf{n}$ & $\%$ & $\mathbf{n}$ & $\%$ & $\mathbf{n}$ & $\%$ & $\mathbf{n}$ & $\%$ \\
\hline Femenino & 3 & 42,9 & 10 & 83,3 & 7 & 53,8 & 3 & 27,3 & 23 & 53,5 \\
\hline Masculino & 4 & 57,1 & 2 & 16,7 & 6 & 46,2 & 8 & 72,7 & 20 & 46,5 \\
\hline Total & 7 & 100,0 & 12 & 100,0 & 13 & 100,0 & 11 & 100,0 & 43 & 100,0 \\
\hline
\end{tabular}


Los patrones imagenológicos más frecuentes para cada tipo de tumor fueron:

- Astrocitoma de bajo grado: hipointenso en T1 $(57,1 \%)$, hiperintenso en T2 (100\%) y FLAIR $(100 \%)$, no presentando realce con el medio de contraste $(100 \%)$, sin restricción en DWI (100\%). Todas fueron lesiones únicas (100\%), de localización intra axial supratentorial $(100 \%)$ y sin edema perilesional $(85,7 \%)$.

- Glioblastoma: isointenso en T1 (58,3\%), variable en T2 (41,7\% isointenso, $41,7 \%$ hiperintenso) y FLAIR (41,7\% isointenso, 50\% hiperintenso), presentando realce con el medio de contraste $(100 \%)$, restricción en DWI $(83,3 \%)$, y en donde la mayoría fueron lesiones únicas $(91,7 \%)$, de localización intra axial supratentorial $(83,3 \%)$ y edema perilesional $(83,3 \%)$.
- Meningioma: isointenso en T1 (76,9\%), hiperintenso en T2 (76,9\%) y FLAIR $(76,9 \%)$, presentando realce con el medio de contraste $(100 \%)$, restricción en DWI (100\%). Todas fueron lesiones únicas $(100 \%)$, la mayoría de localización extra axial supratentorial $(92,3 \%)$ y edema perilesional $(53,8 \%)$.

- Metástasis: isointenso en T1 (63,6\%), hiperintenso en T2 $(81,8 \%)$ y FLAIR $(81,8 \%)$, presentando realce con el medio de contraste $(100 \%)$, restricción en DWI $(63,6 \%)$, lesiones únicas $(63,6 \%)$ y múltiples $(36,4 \%)$, donde la mayoría fueron de localización intra axial supratentorial $(36,4 \%)$, con edema perilesional $(72,7 \%)$ y componente quístico $(9,1 \%)$, (Tabla 6).

Tabla 5. Prevalencia y distribución en el tiempo, de las neoplasias cerebrales en el H.U.C. diagnosticadas por histopatología, a nivel global y por tipo de lesión, $n=43$.

HISTOPATOLOGIA

\begin{tabular}{|c|c|c|c|c|c|c|c|c|c|c|}
\hline & \multicolumn{2}{|c|}{$\begin{array}{l}\text { Astrocitoma de bajo } \\
\text { grado }\end{array}$} & \multicolumn{2}{|c|}{ Glioblastoma } & \multicolumn{2}{|c|}{ Meningioma } & \multicolumn{2}{|c|}{ Metástasis } & \multicolumn{2}{|c|}{ Total } \\
\hline AÑO & $\mathbf{n}$ & $\%$ & n & $\%$ & $\mathbf{n}$ & $\%$ & n & $\%$ & n & $\%$ \\
\hline 2013 & 0 & 0,0 & 2 & 50,0 & 1 & 25,0 & 1 & 25,0 & 4 & 100,0 \\
\hline 2014 & 4 & 40,0 & 0 & 0,0 & 4 & 40,0 & 2 & 20,0 & 10 & 100,0 \\
\hline 2015 & 1 & 11,1 & 5 & 55,6 & 1 & 11,1 & 2 & 22,2 & 9 & 100,0 \\
\hline 2016 & 1 & 12,5 & 3 & 37,5 & 3 & 37,5 & 1 & 12,5 & 8 & 100,0 \\
\hline 2017 & 0 & 0,0 & 2 & 22,2 & 2 & 22,2 & 5 & 55,6 & 9 & 100,0 \\
\hline 2018 & 1 & 33,3 & 0 & 0,0 & 2 & 66,7 & 0 & 0,0 & 3 & 100,0 \\
\hline Total & 7 & 16,3 & 12 & 27,9 & 13 & 30,2 & 11 & 25,6 & 43 & 100,0 \\
\hline
\end{tabular}


Tabla 6. Patrones imagenológicos predominantes en la RM vs diagnóstico histopatológico de las neoplasias en los pacientes objeto de estudio, $n=43$

\begin{tabular}{|c|c|c|c|c|c|c|c|c|c|}
\hline & \multicolumn{9}{|c|}{ DIAGNOSTICO HISTOPATOLÓGICO } \\
\hline & \multicolumn{2}{|c|}{$\begin{array}{c}\text { Astrocitoma } \\
\mathrm{n}=7\end{array}$} & \multicolumn{2}{|c|}{$\begin{array}{c}\text { Glioblastoma } \\
\quad \mathrm{n}=12\end{array}$} & \multicolumn{2}{|c|}{$\begin{array}{l}\text { Meningioma } \\
\quad \mathrm{n}=13\end{array}$} & \multicolumn{2}{|c|}{$\begin{array}{l}\text { Metástasis } \\
\mathrm{n}=\mathbf{1 1}\end{array}$} & \multirow[t]{2}{*}{$\mathbf{p}$} \\
\hline & $\mathbf{n}$ & $\%$ & $\mathbf{n}$ & $\%$ & $\mathbf{n}$ & $\%$ & $\mathbf{n}$ & $\%$ & \\
\hline & \multicolumn{9}{|c|}{ Comportamiento en $\mathrm{T} 1$} \\
\hline Hiperintenso & 0 & 0,0 & 0 & 0,0 & 0 & 0,0 & 1 & 9,1 & \multirow{3}{*}{0,462} \\
\hline Hipointenso & 4 & 57,1 & 5 & 41,7 & 3 & 23,1 & 3 & 27,3 & \\
\hline Isointenso & 3 & 42,9 & 7 & 58,3 & 10 & 76,9 & 7 & 63,6 & \\
\hline \multicolumn{10}{|l|}{ Comportamiento en T2 } \\
\hline Hiperintenso & 7 & 100,0 & 5 & 41,7 & 10,0 & 76,9 & 9,0 & 81,8 & \multirow{3}{*}{0,084} \\
\hline Hipointenso & 0 & 0,0 & 2 & 16,7 & 0,0 & 0,0 & 0,0 & 0,0 & \\
\hline Isointenso & 0 & 0,0 & 5 & 41,7 & 3,0 & 23,1 & 2,0 & 18,2 & \\
\hline \multicolumn{10}{|l|}{ Comportamiento en FLAIR } \\
\hline Hiperintenso & 7 & 100,0 & 6 & 50,0 & 10,0 & 76,9 & 9,0 & 81,8 & \multirow{3}{*}{0,257} \\
\hline Hipointenso & 0 & 0,0 & 1 & 8,3 & 0,0 & 0,0 & 0,0 & 0,0 & \\
\hline Isointenso & 0 & 0,0 & 5 & 41,7 & 3,0 & 23,1 & 2,0 & 18,2 & \\
\hline \multicolumn{10}{|l|}{$\begin{array}{l}\text { Comportamiento con medio de } \\
\text { contraste }\end{array}$} \\
\hline No realce & 7 & 100,0 & 0 & 0,0 & 0 & 0,0 & 0 & 0,0 & \multirow[b]{2}{*}{$<0,05$} \\
\hline Realce & 0 & 0,0 & 12 & 100 , & 13 & 100 & 11 & 100 & \\
\hline \multicolumn{10}{|l|}{ Comportamiento en DWI } \\
\hline No restringe & 7 & 100,0 & 2 & 16,7 & 0 & 0,0 & 4 & 36,4 & \multirow[b]{2}{*}{$<0,05$} \\
\hline Restringe & 0 & 0,0 & 10 & 83,3 & 13 & 100 & 7 & 63,6 & \\
\hline \multicolumn{10}{|l|}{ Cantidad de lesiones } \\
\hline Múltiples & 0 & 0,0 & 1 & 8,3 & 0 & 0,0 & 4 & 36,4 & \multirow[b]{2}{*}{$<0,05$} \\
\hline Única & 7 & 100,0 & 11 & 91,7 & 13 & 100 & 7 & 63,6 & \\
\hline \multicolumn{10}{|l|}{ Edema perilesional } \\
\hline No & 6 & 85,7 & 2 & 16,7 & 6 & 46,2 & 3 & 27,3 & \multirow[b]{2}{*}{$<0,05$} \\
\hline $\mathrm{Si}$ & 1 & 14,3 & 10 & 83,3 & 7 & 53,8 & 8 & 72,7 & \\
\hline \multicolumn{10}{|l|}{ Componente quístico } \\
\hline No & 7 & 100,0 & 12 & 100 & 13 & 100 & 10 & 90,9 & 0,395 \\
\hline $\mathrm{Si}$ & 0 & 0,0 & 0 & 0,0 & 0 & 0,0 & 1 & 9,1 & \\
\hline \multicolumn{10}{|l|}{ Presencia de calcificaciones } \\
\hline No & 7 & 100 & 12 & 100 & 13 & 100 & 11 & 100 & \\
\hline $\mathrm{Si}$ & 0 & 0,0 & 0 & 0,0 & 0 & 0,0 & 0 & 0,0 & \\
\hline \multicolumn{10}{|l|}{ Localización de la lesión } \\
\hline Extra axial-bilateral & 0 & 0,0 & 0 & 0,0 & 0 & 0,0 & 1 & 9,1 & \\
\hline Extra axial-infratentorial & 0 & 0,0 & 0 & 0,0 & 1 & 7,7 & 2 & 18,2 & $<0,05$ \\
\hline Extra axial-supratentorial & 0 & 0,0 & 2 & 16,7 & 12 & 92,3 & 0 & 0,0 & \\
\hline Intra axial-bilateral & 0 & 0,0 & 0 & 0,0 & 0 & 0,0 & 2 & 18,2 & \\
\hline Intra axial-infratentorial & 0 & 0,0 & 0 & 0,0 & 0 & 0,0 & 2 & 18,2 & \\
\hline Intra axial-supratentorial & 7 & 100,0 & 10 & 83,3 & 0 & 0,0 & 4 & 36,4 & \\
\hline
\end{tabular}

Al aplicar la prueba de Chi cuadrado se encontraron diferencias estadísticamente significativas con valores de $\mathrm{p}<0,05$ para: el comportamiento de las lesiones en DWI y con el medio de contraste, la cantidad de lesiones, la presencia de edema perilesional y la localización de las mismas.
Al comparar los resultados de la RM con la histopatología se identificó que la resonancia fue más sensible (100\%) y con un VPP más alto $(100 \%)$ en los casos de meningioma y astrocitoma de bajo grado, mientras que fue muy específica $(100 \%) \mathrm{y}$ con VPN de $100 \%$ para el glioblastoma. En el caso del índice de exactitud encontramos que la RM fue 
más exacta $(97,6 \%)$ para los astrocitomas de bajo grado $y$ en el menor grado $(83,7 \%)$ para las metástasis. En todos los casos se aplicó la prueba exacta de Fisher y se encontró que hubo diferencias estadísticamente significativas entre ambos métodos diagnósticos $(\mathrm{p}<0,05)($ Tablas 7 y 8$)$.

Tabla 7. Diagnóstico imagenológico vs diagnóstico histopatológico de las neoplasias encontradas en los pacientes objeto de estudio, $n=43$ HISTOPATOLOGIA

\begin{tabular}{|c|c|c|c|c|c|c|c|c|c|c|}
\hline \multirow[b]{2}{*}{$\begin{array}{l}\text { DIAGNOSTICO } \\
\text { IMAGENOLOGICO }\end{array}$} & \multicolumn{2}{|c|}{$\begin{array}{l}\text { Astrocitoma de bajo } \\
\text { grado } n=7\end{array}$} & \multicolumn{2}{|c|}{$\begin{array}{c}\text { Glioblastoma } \\
n=12\end{array}$} & \multicolumn{2}{|c|}{$\begin{array}{c}\text { Meningioma } \\
n=13\end{array}$} & \multicolumn{2}{|c|}{$\begin{array}{c}\text { Metástasis } \\
n=13\end{array}$} & \multicolumn{2}{|c|}{$\begin{array}{l}\text { Total } \\
\mathrm{n}=43\end{array}$} \\
\hline & $\mathbf{n}$ & $\%$ & $\mathbf{n}$ & $\%$ & $\mathbf{n}$ & $\%$ & $\mathbf{n}$ & $\%$ & $\mathbf{n}$ & $\%$ \\
\hline Astrocitoma de bajo grado & 7 & 100,0 & 1 & 8,3 & 0 & 0,0 & 0 & 0,0 & 8 & 18,6 \\
\hline Glioblastoma & 0 & 0,0 & 6 & 50,0 & 0 & 0,0 & 0 & 0,0 & 6 & 14,0 \\
\hline Metástasis & 0 & 0,0 & 3 & 25,0 & 0 & 0,0 & 7 & 63,6 & 10 & 23,3 \\
\hline
\end{tabular}

Tabla 8. Sensibilidad, especificidad, VPP, VPN, exactitud global y cociente de probabilidad positivo y negativo de la RM en comparación con la histopatología.

\begin{tabular}{lcccc}
\hline & DIAGNÓSTICO HISTOPATOLÓGICO & & \\
& Meningioma & Astrocitoma & Glioblastoma & Metástasis \\
\hline RESONANCIA MAGNÉTICA & & & & \\
\hline Sensibilidad & $100,0 \%$ & $100,0 \%$ & $50,0 \%$ & $63,6 \%$ \\
\hline Especificidad & $86,7 \%$ & $97,2 \%$ & $100,0 \%$ & $90,6 \%$ \\
\hline VPP & $76,4 \%$ & $87,5 \%$ & $100,0 \%$ & $70,0 \%$ \\
\hline VPN & $100,0 \%$ & $100,0 \%$ & $83,7 \%$ & $87,8 \%$ \\
\hline Exactitud global & $90,7 \%$ & $97,6 \%$ & $86,1 \%$ & $83,7 \%$ \\
\hline Cociente de probabilidad positivo & 7,5 & 35,7 & 0 & 6,7 \\
\hline Cociente de probabilidad negativo & 0 & 0 & 0,5 & 0,4 \\
\hline Valor de p & $<0,05$ & $<0,05$ & $<0,05$ & $<0,05$ \\
\hline
\end{tabular}

\section{DISCUSIÓN}

En el presente estudio se obtuvo una muestra pequeña a pesar de haber incluido varios años, esto es debido a que el H.U.C. ser un centro de referencia en la región, no es la institución más importante para el tratamiento de patologías neuroquirúrgicas. Casi la totalidad de los casos provenían del departamento de Bolívar, esto dado que se realizó la recolección de datos en un hospital público de referencia de la ciudad de Cartagena.

La mayoría de los casos que se presentaron ocurrieron en el sexo femenino, lo cual está de acuerdo con otros estudios al respecto de esta patología (14). Es de resaltar que en una alta frecuencia los tumores fueron glioblastomas y esto no es concordante con esas publicaciones; sin embargo, en este estudio no se encontraron diferencias significativas entre el género y el tipo histológico de tumor y esto puede deberse al tamaño de la muestra.

Los síntomas de inicio predominantes fueron cefalea y convulsiones lo cual está de acuerdo con 1o reportado por otros autores $(15,16)$. En este estudio no hubo diferencias estadísticas entre el tipo de tumor y las manifestaciones clínicas y esto se puede deber a que los síntomas anteriormente mencionados son generales a la mayoría de los tumores del SNC. El tiempo transcurrido entre la presentación de los síntomas y la consulta al H.U.C. fue variable, como es de esperarse, la mayoría de los pacientes consultaron rápidamente (menos de 30 días), sin embargo, un grupo importante tardaron más de lo esperado $\mathrm{y}$ esto demora en los trámites 
administrativos en sus respectivas empresas de salud.

En cuanto a la frecuencia por tipo histológico de tumor hubo una mayor proporción de meningiomas seguido de glioblastomas y de lesiones metastásicas, contrasta con otras publicaciones $(17,18)$ en las que las lesiones metastásicas son el tipo más frecuente cuando se evalúa el conjunto de tumores primarios y secundarios del SNC, y podría ser debido a que generalmente al momento del diagnóstico de éstas, el paciente tiene un gran compromiso en su estado general y no alcanza a ser llevado a la toma de biopsia. Hablando estrictamente de los tumores primarios del SNC su frecuencia en el H.U.C. concuerda con lo reportado en otros estudios (14).

En esta investigación se encontró que al igual que en trabajos reportados anteriormente $(14,17,18)$, existen diferencias significativas entre el tipo de tumor y la edad, identificándose un incremento en el número de tumores cerebrales a medida que aumenta la edad en especial en el caso de los glioblastomas, meningiomas y las metástasis.

Entre los años 2014 a 2017 se presentaron acumulados totales de casos similares, mientras que en el 2013 y 2018 el número fue menor, esto debido a que no se incluyeron los años completos sino un trimestre de cada uno de ellos. Es de anotar que el hecho de encontrar mayor frecuencia de meningioma durante el periodo de estudio está de acuerdo con lo publicado (14), en donde el meningioma es el tipo histopatológico más frecuente de los tumores primarios del SNC.

Los patrones imagenológicos más frecuentes que se encontraron en la RM para todos los tipos histopatológicos de tumores del SNC evaluados, concuerdan con los descritos en la literatura (7). En este estudio se encontraron diferencias estadísticas entre varias características imagenológicas y el tipo histológico, como es el caso del comportamiento de las lesiones con el medio de contraste y en las secuencias DWI, lo cual coincide con otras publicaciones $(7,19,20)$.

Al analizar los resultados de la RM encontramos que en dos de los casos no se identificaron lesiones tumorales, a pesar de esto, a los pacientes se les realizó biopsia y el resultado histopatológico comprobó la presencia de tumor, por lo cual se hace importante tener una retroalimentación desde el grupo de patología a los radiólogos a fin de encontrar las posibles causas del error diagnóstico.

Teniendo en cuenta la sensibilidad, especificidad y los valores predictivos podemos decir que ante un resultado negativo para meningioma o astrocitoma de bajo grado en la RM seguramente no se encontrarán estas en el resultado de la histopatología; sin embargo, en el caso contrario se pueden presentar algunos falsos positivos. Lo anterior indica que los radiólogos del H.U.C. conocen.

En el caso del glioblastoma, esto sugiere que el radiólogo no está bien familiarizado con los patrones que puede presentar este tipo de tumor en la RM. De manera similar ocurre con las metástasis en la que la sensibilidad es baja comparada con la especificidad y los valores predictivos se encuentran por debajo del $90 \%$, por lo tanto, es necesaria la capacitación continua por parte del grupo de radiólogos y la retroalimentación con el grupo de patología para mejorar la certeza diagnóstica de modo que se apunte a mejorar la sensibilidad en el diagnóstico. En general, la RM presentó buenos índices de exactitud diagnóstica.

En conclusión, en el H.U.C. la RM presenta buen desempeño en el diagnóstico de tumores cerebrales, en especial los astrocitomas de bajo grado y los meningiomas, $\mathrm{y}$ en menor grado para los glioblastomas y las lesiones metastásicas. Los hallazgos en cuanto a características generales de los pacientes con tumores cerebrales y sus presentaciones imagenológicas coincidieron con lo reportado en otros lugares del mundo. 


\section{REFERENCIAS}

1. Organizacion Mundial de la Salud. [Internet]. Phoenix: OMS; 2017 [citado 2017 marzo 20]. Centro de prensa; [aprox. 6 pantallas]. Disponible en: http:/www.who.int/mediacentre/factsheets/fs297/es/

2. National Cancer Institute. [Internet]. Frederick: The Institute; 2014 [citado 2017 marzo 23]. Cancer Statistics; [aprox. 7 pantallas]. Disponible en: https://seer.cancer.gov/statfacts/html/brain.html

3. Pardo C, Cendales R. Incidencia, mortalidad y prevalencia de cáncer en Colombia, 2007-2011. Primera edición. Bogotá. D.C. Instituto Nacional de Cancerología, 2015, v.1. p. 148

4. MINSALUD. [Internet]. Bogotá. D.C: MINSALUD; 2017 [citado 2017 marzo 23]. Plan Decenal-Análisis de la Situación en Salud Cartagena; [aprox. 137 pantallas]. Disponible en: https://www.minsalud.gov.co/plandecenal/mapa/Analisi s-de-Situacion-Salud-Cartagena-2011.pdf

5. Ramos E, Tunón M, Rivas F, Veloza L. Tumores primarios del sistema nervioso central en Cartagena, 2001- 2006. Rev salud pública. 2010 Mar; 12(2): p. 25767.

6. Louis DN, Perry A, Reifenberger G, von Deimling A, Figarella-Branger D, Cavenee WK, et al. The 2016 World Health Organization Classification of Tumors of the Central Nervous System: a summary. Acta Neuropathol. 2016 Jun; 131(6): p. 803-20.

7. Chourmouzi D, Papadopoulou E, Marias K, Drevelegas A. Imaging of Brain Tumors. Surg Oncol Clin N Am. 2014 Aug; 23(4): p. 629-84.

8. The Royal College of Radiologists. Quality assurance in radiology reporting: peer feedback. London: RCR; 2014.

9. Noguchi T, Yoshiura T, Hiwatashi A, Togao O, Yamashita K, Nagao E. Perfusion Imaging of Brain Tumors Using Arterial Spin-Labeling: Correlation with Histopathologic Vascular Density. AJNR. 2008 Apr; 29 (4): p. 688-93.

10. Rodríguez M, Villafuerte $\mathrm{D}$, Conde T, Díaz O, Martínez A. Caracterización tomográfica e histológica de las neoplasias intracraneales. Medisur. 2010 Mar; 8(2): p. 9-14.
11. Saldívar C, Guerrero G, Benítez M, Reyes M. Utilidad de la resonancia magnética en el diagnóstico y clasificación de los tumores astrocíticos. Anales de Radiología México. 2016 Oct; 15(4): p. 279-93.

12. Páez A, Burbano N, Merchancano C, Erazo N, Muñoz A. Caracterización de los tumores cerebrales en un Hospital Universitario de Pereira, Colombia: un estudio retrospectivo. Rev. Méd. Risaralda. 2013 Mar; 19 (2): p. 120-25.

13. Vega Molina, Alejandro. Caracterización clínica e imagenológica de pacientes con glioblastoma o astrocitoma anaplásico atendidos en el Instituto Nacional de Cancerología durante el periodo enero 2007 diciembre 2013. Repositorio Institucional Biblioteca Universidad Nacional de Colombia. 2016. Disponible en: http://www.bdigital.unal.edu.co/51133/

14. Ostrom Q, Gittleman H, Liao P, Vecchione-Koval T, Wolinsky Y, Kruchko C, et al. CBTRUS Statistical Report: Primary brain and other central nervous system tumors diagnosed in the United States in 2010-2014. Neuro Oncol. 2017 Nov; 19 (S5): p. 1-88.

15. Grant R. Overview: brain tumour diagnosis and management/royal college of physicians guidelines. J Neurol Neurosurg Psychiatry. 2004 Jun; 75 (S2): ii18ii23.

16. Ivan Comelli, Giuseppe Lippi, Valentina Campana, Franco Servadei, Gianfranco Cervellin. Clinical presentation and epidemiology of brain tumors firstly diagnosed in adults in the Emergency Department: a 10-year, single center retrospective study. Ann Transl Med. 2017 Jul; 5 (13): 269.

17. Contreras L. Epidemiología de tumores cerebrales. Rev Med Clin Condes. 2017 May; 28 (3): p. 332-338.

18. Ostrom QT, Wright CH, Barnholtz-Sloan JS. Brain metastases: epidemiology. Handb Clin Neurol. 2018;149: p. 27-42.

19. Batash R, Asna N, Schaffer P, Francis N, Schaffer M. Glioblastoma Multiforme, Diagnosis and Treatment; Recent Literature Review. Curr Med Chem. 2017 May.

20. Boyd A, Smirniotopoulos J, Horkanyne-Szakaly I. Intraventricular Neoplasms: Radiologic-Pathologic Correlation. Radiographics. 2013 Jan; 33(1): p. 21-43. 